\title{
Imaging Approach to Malignant Bone Tumors
}

\section{Subbarao K}

Padmshri Prof. Dr. Kakarla Subbarao, Hyderabad, India

\section{Introduction}

Primary malignant bone tumors are quite rare, whereas metastatic disease to the bone is common. For a number of years, conventional radiology played a major role in the diagnosis. With the advanced imaging techniques ultrasound, color Doppler, MDCT, MRI and PET-CT scans (Tables I \& II), early diagnosis, extent of the disease, staging of the disease and follow up imaging do help a great deal.

\section{C.T. in Malignant Bone Lesions - Table I}

- Cross sectional images

- Soft tissues

- Early calcifications

- Tissue characteristics

- Medullary cavity

- Vessels \& nerves

Role of M.R.I - Table II

- Transitional zone

- Skip areas

- Tissue characteristics

- Soft tissues - necrosis, hemorrhage

- Marrow involvement

- Neurovascular bundles

- M.R. Angiography

Team work involving the orthopedic surgeon, oncologist, pathologist and imageologist goes a long way in dealing with malignant tumors. A modified WHO classification is listed in table III.

\section{Primary Malignant bone tumours} (modified who classification)

\section{Table III}

- Bone Forming - Osteosarcoma \& Variants, Ewing's

- Cart. Forming - Chondrosarcoma

- Marrow Tumours Multi.myeloma,

- Lympho-sarcoma, leukemia

- Fibrous Tissue - Fibrosaroma, Malignant fibrous Histiocytoma

- Giant Cell - Mgt. Giant Cell Tumour

- Vascular - Angiosarcoma, kaposi's sarcoma

- Miscellaneous -Neurosarcoma, Chordoma, Adamantinoma

A mnemonic is prepared to study the criteria of malignancy, namely STAMPS. S-stands of the site whether epiphysis, metaphysis or diaphysis. T-stands for transitional zone that is the border between normal and abnormal bone. A-stands for age of the patients and aggressive nature of the lesion. M-stands for matrix, whether that is lucent, or calcified or ossified. Ossified matrix is usually cloudy whereas cartilaginous calcification shows punctate, arc like or circular. Vascular matrix may suggest hemangioma. Fatty matrix indicates lipomatous tumor. Pstands for periosteal reaction which can be linear, multilaminated, spiculated, velvety type or presence of Codman's triangle. In benign lesions the periosteal reaction is generally unilaminar. Multilaminar 
periosteal reaction is often encountered in Ewing's sarcoma. Spiculated or Sunburst type of periosteal reaction is noted both in Ewing's sarcoma and osteosarcoma. Sstands for soft tissue swelling and associated calcifications. This is listed in table IV

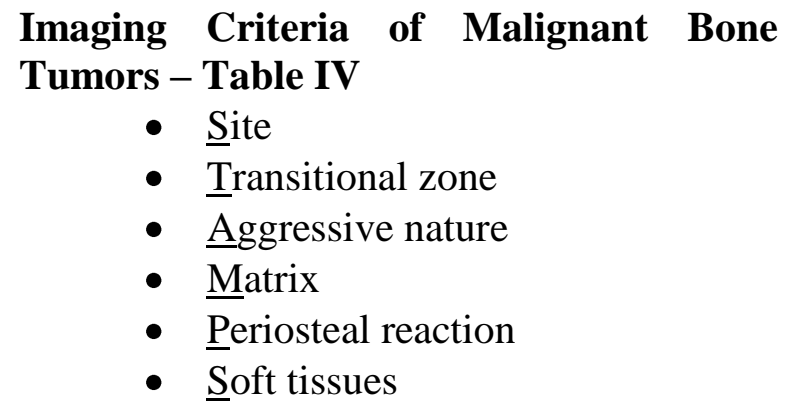

Primary malignant tumors such as Ewing's sarcoma, osteosarcoma and lymphosarcoma are common in younger age group. Fibrosarcomas and chondrosarcoma occur in adults whereas myeloma and metastasis usually occur in older people. Table V

\section{Primary Malignant Bone Tumors}

\section{- Table V}

- Ewing's sarcoma \& Ewing's family of tumors

- Osteosarcoma - Several varieties

- Chondrosarcoma - Several varieties

- Lymphoma - Leukemia

- Fibrosarcoma, malignant fibrous histiocytoma

- Plasmacytoma - Multiple myeloma Poems syndrome

Other primary tumors include hemangioendothelioma, angiosarcoma, Kaposi's sarcoma, rhabdomyosarcoma, liposarcoma etc.

\section{Ewing's sarcoma}

The age ranges between 3-30yrs. Slight predominance in males is noted, about $50 \%$ of them occur in diaphysis of long bones. The transitional zone is wide and the matrix may be ossified. The Saucerization of cortex is one of the typical radiological findings (fig. 1). The periosteal reaction is onion peel in most of the cases (fig. 2). However, spiculated, sunburst and Codman's triangles of periosteal reaction may be seen. Moth eaten or gross lytic areas may be noted. The soft tissue swelling is pronounced when it occurs in the flat bones such as scapula, ileum etc. The sclerosis may be prominent and when it occurs in the vertebral body it is called ivory vertebra (fig. 3). These are listed in table VI

\section{Radiological features - Table VI}

- Common in long bones - diaphysis

- Permeative destructive lytic pattern in medulla

- Wide zone of transition

- Saucerization of cortex

- Lamellar type of periosteal reaction

- Onion peel appearance

- Flat bones permeative destruction with large soft tissue swelling

- Ivory vertebra

- Vertebra plana

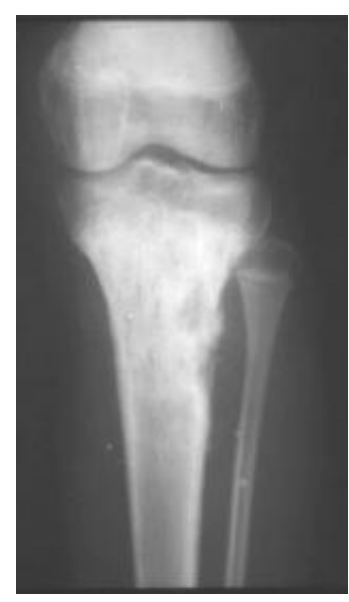

Fig 1: Ewing's sarcoma of diametaphysis of tibia with sclerosis simulating osteosarcoma. However, the saucerization of the cortex is typical of Ewing's sarcoma. 


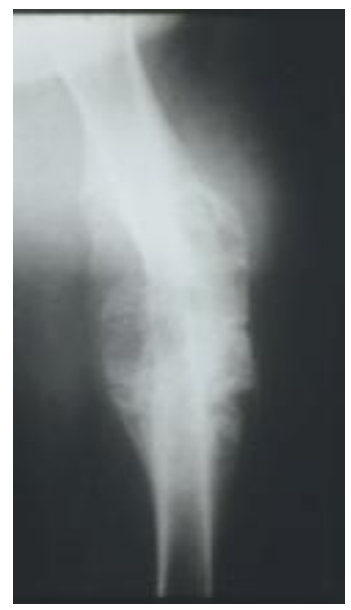

Fig. 2: Ewing's sarcoma of femoral shaft in a $10 \mathrm{yr}$ old boy. Note the Codman's triangles and periosteal reaction.

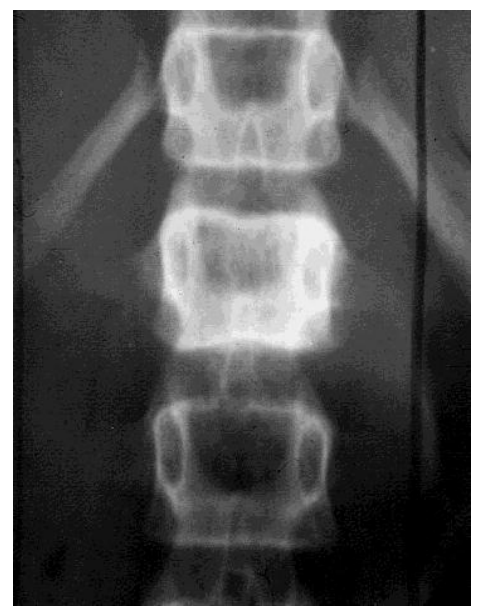

Fig. 3: Ewing's sarcoma of L1 - Ivory vertebra

Plain films are helpful in noting the types of periosteal reaction in the long bones. However, in flat and short bones periosteal reaction is not appreciable. Soft tissue swelling is large and is better identified on MRI and CT (fig. 4). Necrotic areas are also well seen in the advanced imaging techniques.

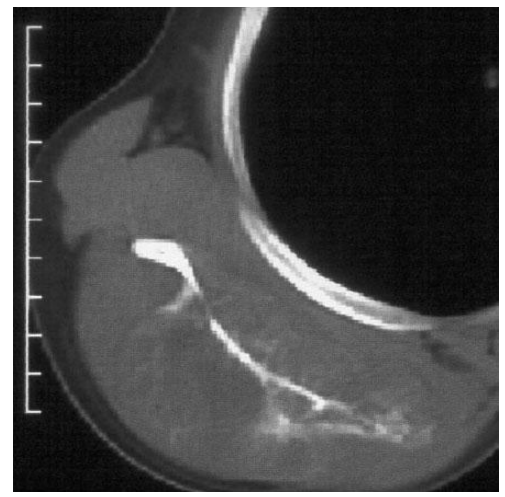

Fig. 4: Ewing's sarcoma CT. Note the soft tissue swelling and spiculated periosteal reaction.

Metastasis from Ewing's sarcoma often occurs in the bones and lungs. There is a group of Ewing sarcoma family of tumors which includes Askin tumor, PNET (Primitive Neuroectodermal Tumor, soft tissue Ewing's sarcoma etc.)

\section{Osteosarcoma - Several varieties}

Several varieties of osteosarcoma exist. Osteogenic sarcoma radiologically is metaphyseal in origin with multiple lucent areas, cortical brake and spiculated periosteal reaction. A large soft tissue mass is associated. Codman's triangle is often present. Extensive pluffy type of new bone formation is noted. Purely osteolytic type of osteosarcomas are also seen, one of which is telangiectatic osteosarcoma. It is encountered in the second and third decades. This is often very aggressive with multiple lytic areas and minimal new bone formation. Table VII consists of these lesions with osteoid matrix

\section{Osteosarcoma - Osteoid Matrix - Table} VII

- Conventional

- Telangiectatic

- Chondroblastic

- Paraosteal

- Periosteal

- Multicentric

Classic osteosarcoma generally occurs in late $1^{\text {st }}$ and $2^{\text {nd }}$ decades. Male predominance is 
noted. It is metaphyseal and rarely extends to the epiphysis. There is diffuse new bone formation with areas of lysis. The periosteal reaction is spiculated sunburst or Codman's triangles. It is important to identify osteoid matrix which is cloudy and cumuls (fig. 5ab \& 6). Type of osteosarcoma are listed in table VIII
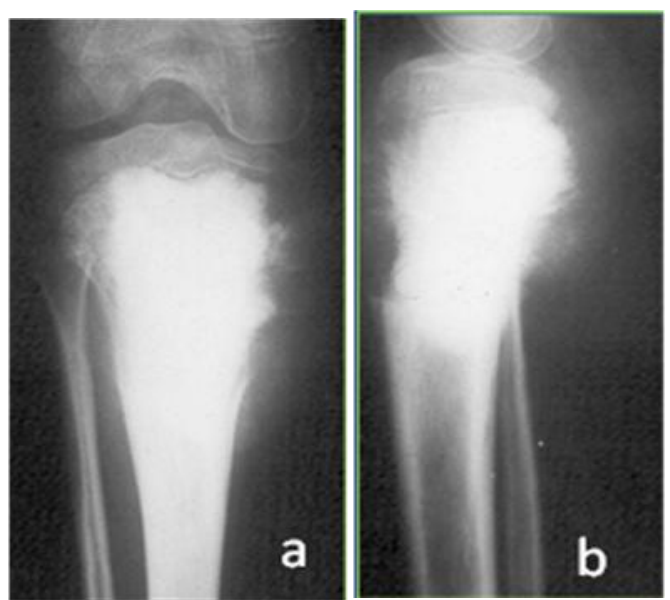

fig. 5ab: Sclerosing type of osteosarcoma in the metaphysis of the tibia. Note the cloud like ossifications and periosteal reaction.

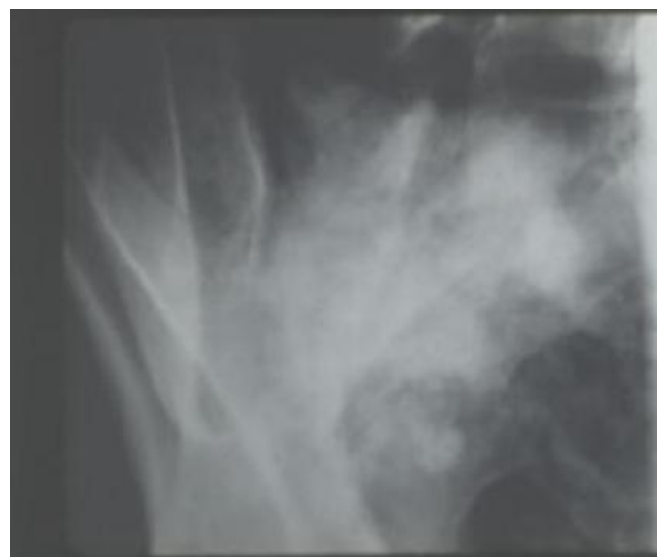

Fig. 6: Osteosarcoma of the sacrum with cloud like matrix

Osteosarcoma - Table VIII

\section{Intramedullary}

- High grade

- Telangiectatic

- Low grade

- Small cell

- Osteosarcomatosis

- Gnathic

\section{Surface}

- Intracortical

- Paraosteal

- Periosteal

- High grade surface

High grade

It is very aggressive with areas of new bone formation and necrosis. Pathological fractures do occur (fig. 7).

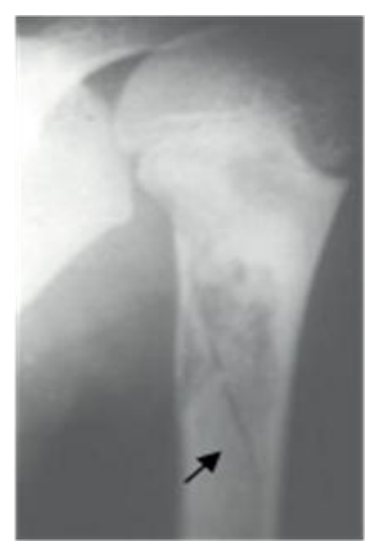

Fig. 7: Lytic type of osteosarcoma with a pathological fracture

\section{Telangiectatic}

This is mostly lytic and not much of new bone or periosteal reaction (fig. 8ab). Occasionally, fluid - fluid levels are seen on MRI.
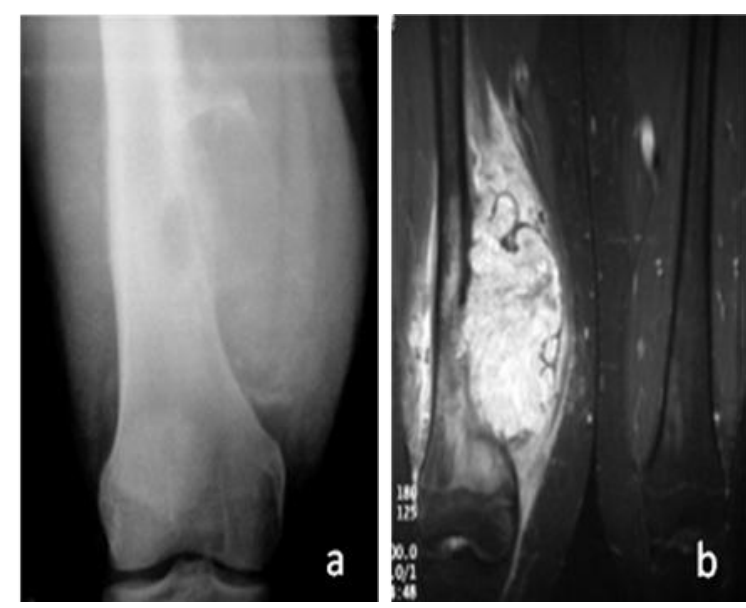

Fig. 8ab: Telangiectatic osteosarcoma lower end of femur. 


\section{Low grade}

Simulates osteoma or osteoblastoma

\section{Small cell}

No definite imaging characters are noted.

Osteosarcoma metastasizes to bones and lungs. MRI reveals the skipped metastasis. Radionuclide scan identifies all the osteoid producing lesions.

\section{Osteosarcomatosis}

There are two verities. One in children and another in adolescence, they are multiple, dense sclerotic areas in the metaphysis as well as in the epiphysis. Sometimes it is mistaken for metastasis from osteosarcoma.

\section{Gnathic}

Osteosarcoma of the mandible is a specific entity. It occurs in $3^{\text {rd }}$ or $4^{\text {th }}$ decade. A mixture of sclerotic and lytic areas are noted. Prognosis is better.

\section{Surface osteosarcoma - Intracortical}

These occur in the diametaphyses of young adults. Multiple layers of periosteal reaction may be noted which are circumscribed (fig. $9 \mathrm{ab}$ ). No other radiological findings are noted.

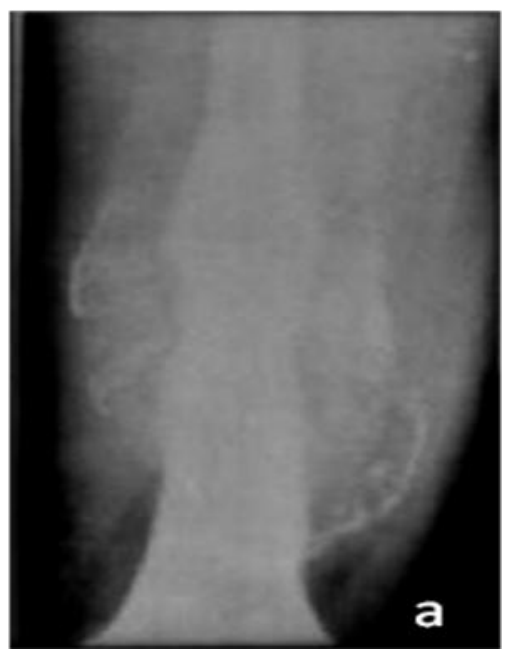

Fig. 9a: Surface osteosarcoma. Note the periosteal reaction \& saucerization

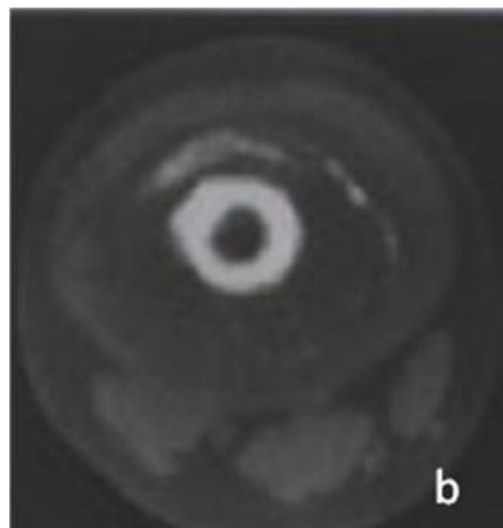

Fig. 9b: Surface osteosarcoma. Note the circumscribed periosteal new bone.

\section{Paraosteal osteosarcoma}

The age is generally at $3^{\text {rd }}$ and $4^{\text {th }}$ decades. There is a long history of pain and swelling. Radiologically a lump of bone occurs in the juxta cortical plane. The peripheral border is lobulated. In some cases it is separated from the host bone by a translucent line. In advanced cases the cortex may be invaded and extend into the medulla. CT is best to identify the involvement of the cortex and medulla. The degree of malignancy is lower than that of classical osteosarcoma (fig. $10 \& 11$ ). 


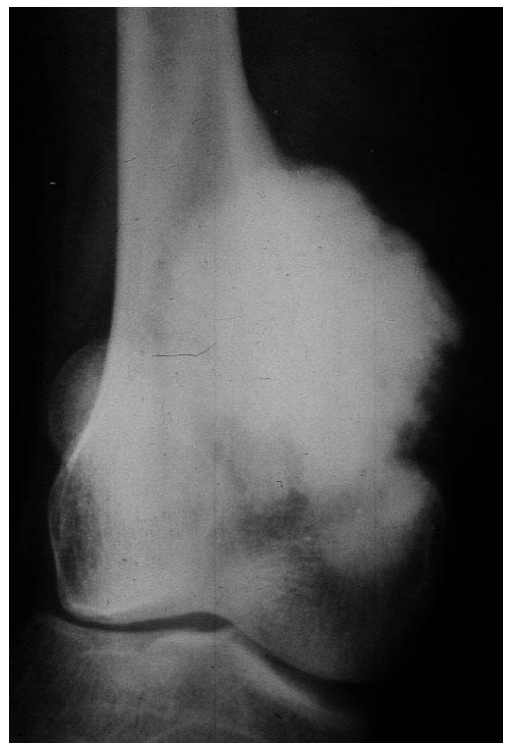

Fig. 10: Paraosteal osteosarcoma arising from the lower end of femur

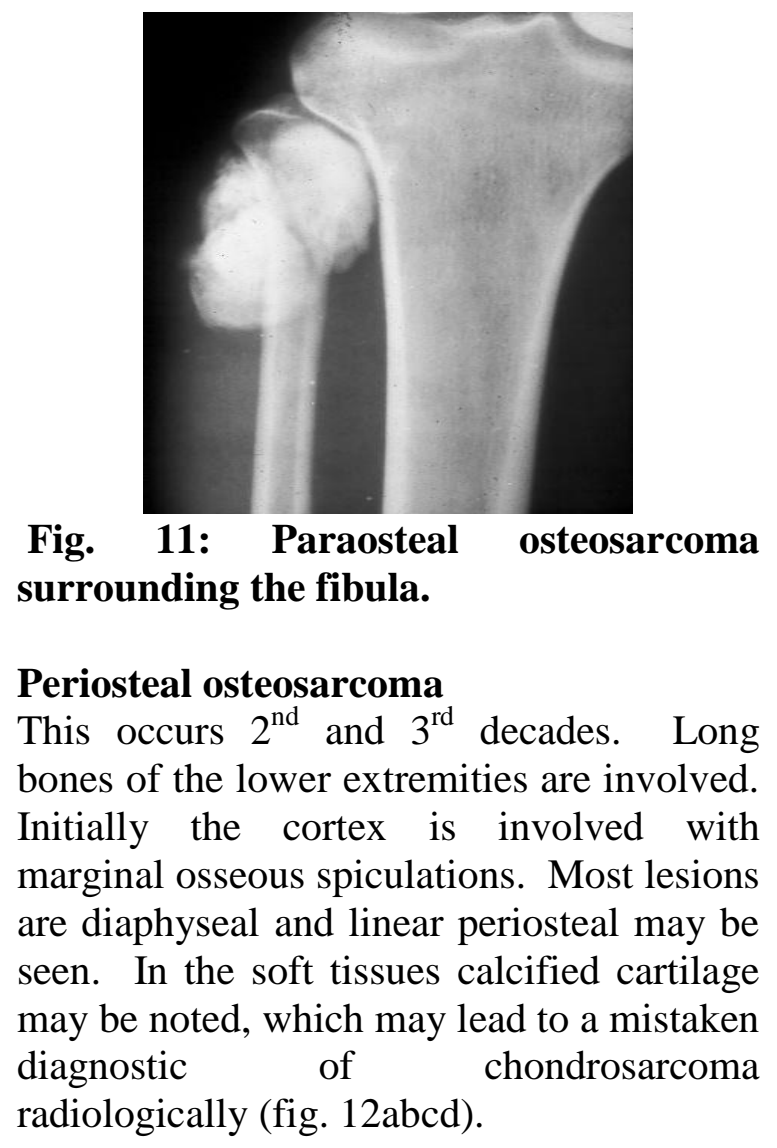

\section{Periosteal osteosarcoma}

This occurs $2^{\text {nd }}$ and $3^{\text {rd }}$ decades. Long bones of the lower extremities are involved. Initially the cortex is involved with marginal osseous spiculations. Most lesions are diaphyseal and linear periosteal may be seen. In the soft tissues calcified cartilage may be noted, which may lead to a mistaken radiologically (fig. 12abcd).
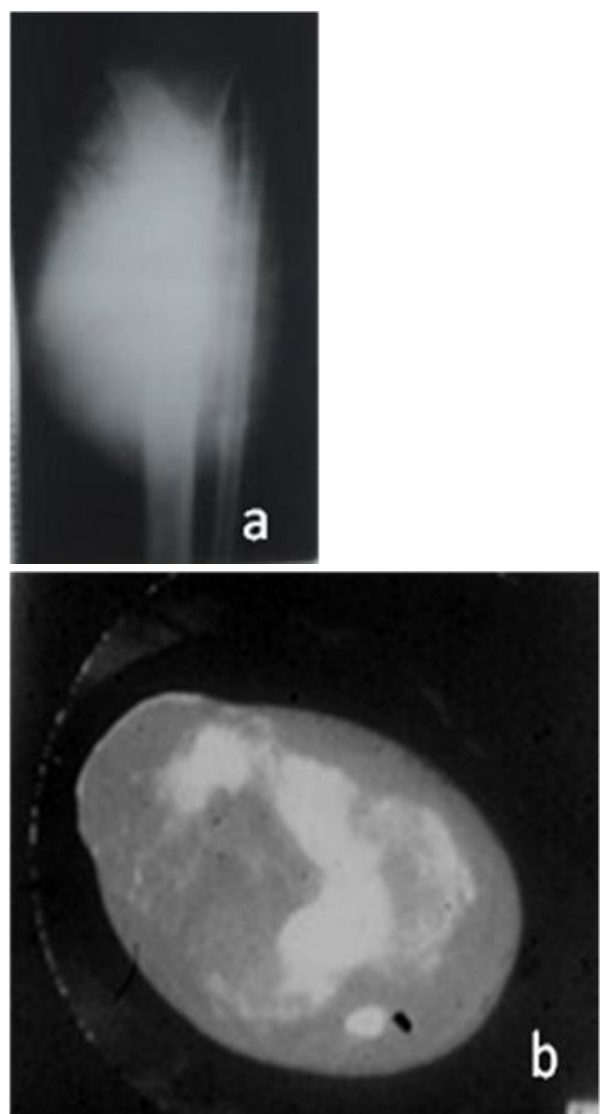

Fig. 12ab: Periosteal osteosarcoma of diaphysis of tibia. CT shows the extent of the tumor.

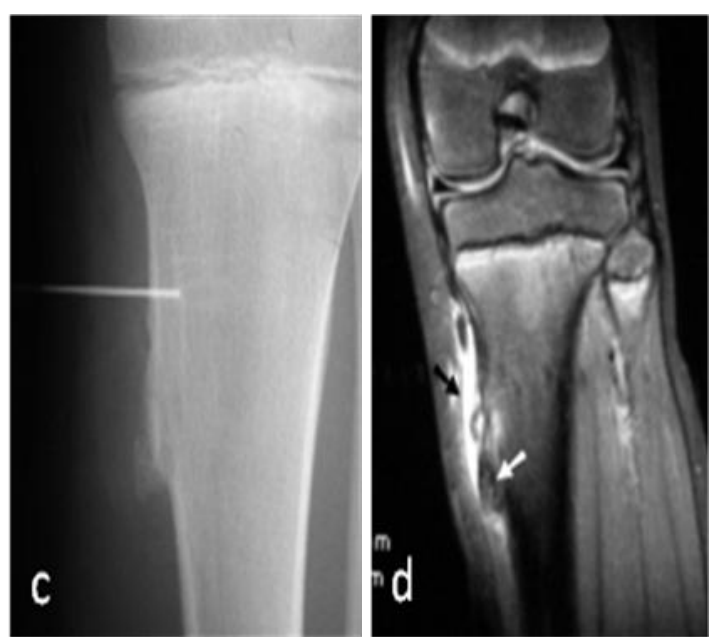

Fig. 12cd: Perioseal osteosarcoma- c. Plain film, d. MRI

\section{Multicentric osteosarcomatosis}

These are often encountered in children with multiple dense lesions in most of the bones. This has to be differentiated from osteoblastic metastasis from osteogenic sarcoma (fig. 13ab) 


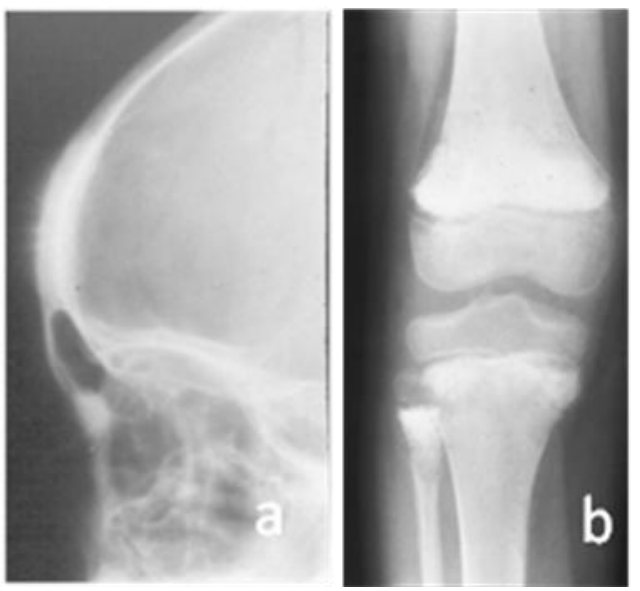

Fig. 13ab: Multicentric osteosarcomatosis in a 5 yr old child. Note the dense lesions around the knee and skull.

\section{Post radiation osteosarcoma}

Secondary osteosarcoma may be encountered subsequent to radiation, particularly following treatment of carcinoma of breast (fig. 14ab).

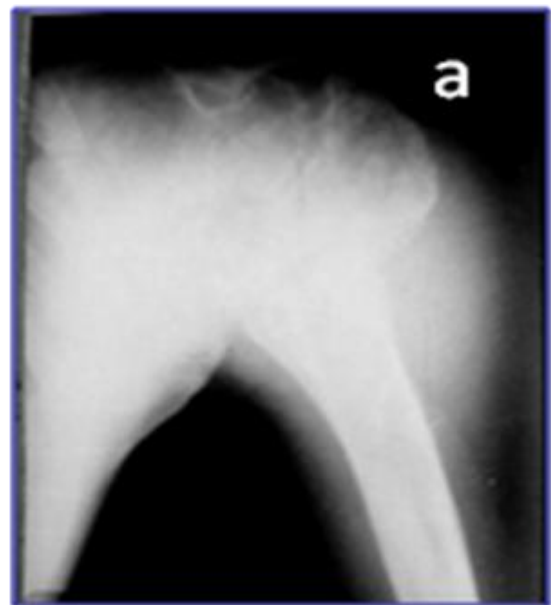

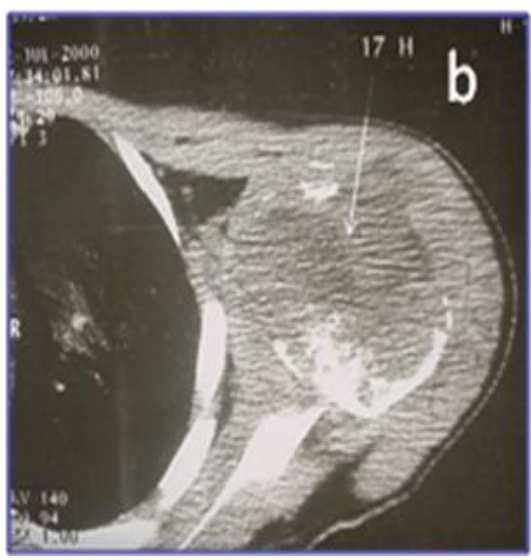

Fig. 14ab: Post radiation osteosarcoma- a. Plain radiography $\&$ b. $\mathrm{CT}$.

Most of the lesions are osteolytic. However, radiation osteitis may be seen in the adjacent bones

Secondary osteosarcoma can occur in Paget's disease or fibrous dysplasia

\section{Primary Chondrosarcoma - Several Varieties}

It is rarely seen in children, the age range is between $4^{\text {th }}$ and $6^{\text {th }}$ decades. The primary chondrosarcoma arises centrally in the medulla. Radiologically, an osteolytic area with smooth scalloped endosteal erosion is seen (fig. 15). The transitional zone may vary. Fusiform thickening of cortex with a velvety periosteal reaction iss present. Central calcifications indicative of cartilaginous origin are present (fig. 16). In chondrosarcoma of the flat bones such as ileum and scapula, a large soft tissue mass is noted with calcifications. CT helps in identifying the mass and the involvement of soft tissue structures. On MRI T2 weighted images show bright signals with lobulations. 


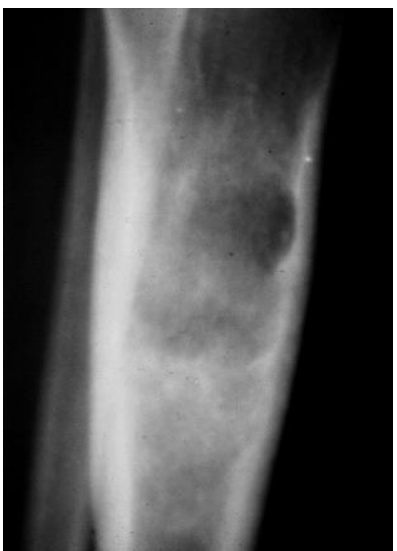

Fig. 15: Chondrosarcoma of the tibia in a 40 yr old man. Note the lytic lesion with endosteal scalloping and thickened cortex.

\section{Juxta cortical chondrosarcoma}

It is encountered in $3^{\text {rd }}$ and $4^{\text {th }}$ decades. Radiologically a large soft tissue mass extends outward from the cortex. Calcifications are noted in the matrix.

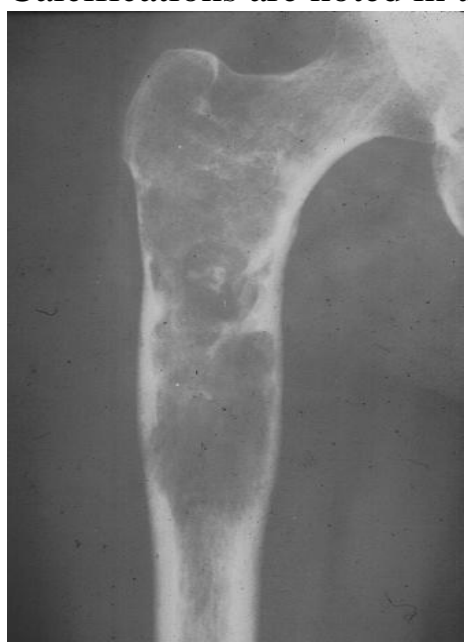

Fig. 16: Chondrosarcoma of the femur with endosteal scalloping and multiple calcifications

\section{De-differentiated Chondrosarcoma}

This is encountered in $5^{\text {th }}$ and $6^{\text {th }}$ decades. This generally occurs in long standing chondroid lesion. Pathological fractures may occur. The large soft tissue mass shows the dedifferentiated portion which is of greater malignancy. Depending upon the site of biopsy, it may show low grade chondrosarcoma, fibrous histiocytoma or osteosarcoma.

\section{Clear cell chondrosarcoma}

It may be encountered in $3^{\text {rd }}$ to $7^{\text {th }}$ decades. The sites of predilection include proximal end of femur and humerus. Radiologically, large osteolytic lesion is noted with a narrow zone of transition. This may extend to epiphysis. Calcification is rare (fig. 17a).

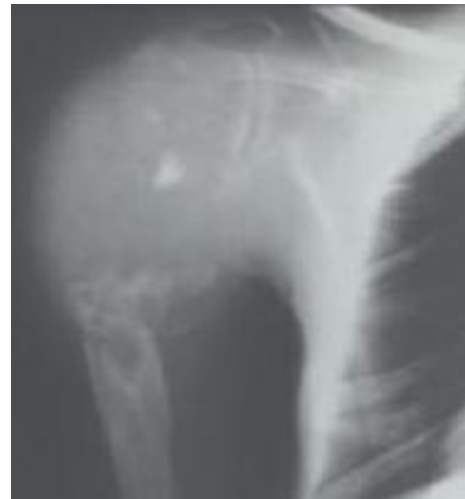

Fig. 17a: Clear Cell chondrosarcoma in the proximal end of humerus extending into the epiphysis.

\section{Mesenchymal chondrosarcoma}

It is encountered in $2^{\text {nd }}$ and $3^{\text {rd }}$ decades $(60 \%)$. Facial bones, ribs and long tubular bones may be affected. Approximately $1 / 3^{\text {rd }}$ of the cases originate in soft tissues. Radiologically, most of the cases show calcifications both in the bone and in the soft tissues (fig. 17b).

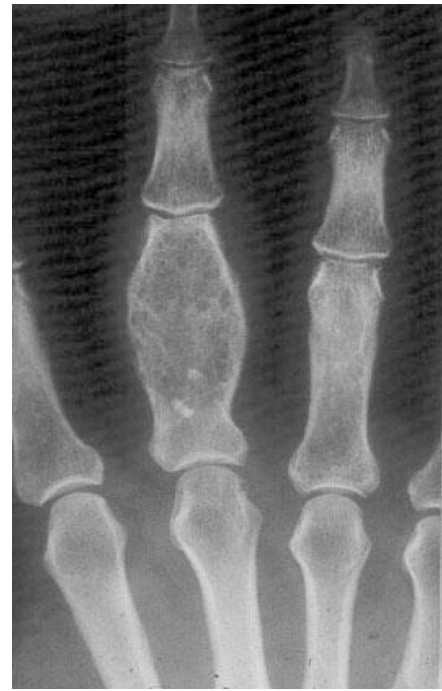

Fig. 17b: Mesenchymal chondrosarcoma in a 32 yr old involving the proximal phalanx of middle finger. 
Secondary chondrosarcoma occurs in $1 \%$ of enchondromas, $10-15 \%$ of hereditary multiple exostoses and 25-30\% of Maffucci syndrome (fig. 18). CT often helps in depicting soft tissue involvement (fig. 19)

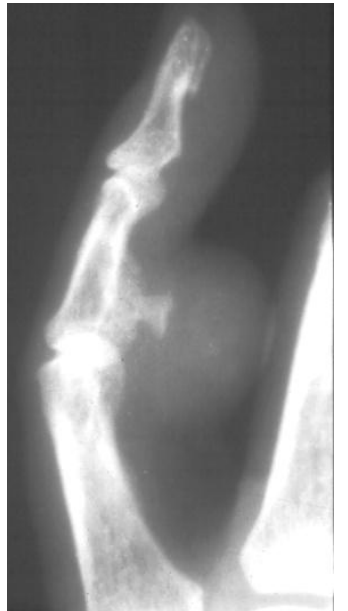

Fig. 18: Chodnrosarcoma in exostosis of middle phalanx of index finger

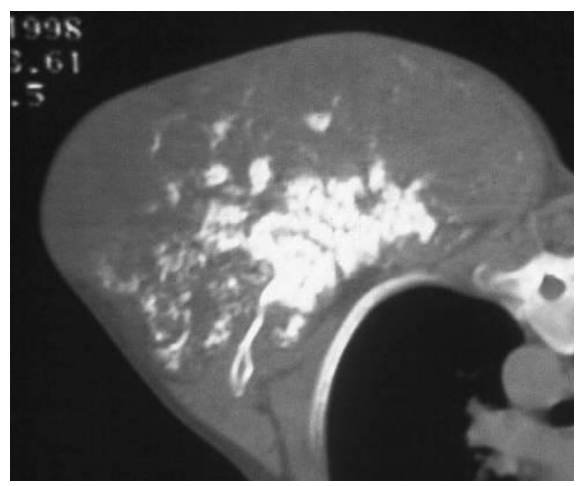

Fig. 19: Secondary chondrosarcoma arising from exostosis of the scapula. Note the scattered calcifications.

On occasion, difficulty arises to note the changes occurring in the transformation of a benign lesion into malignant lesion. The thickness of the cartilage, when it is more than $3 \mathrm{cms}$, malignancy must be considered. Another criterion is the more the scatter of the calcifications the more possibility of malignancy. The third criterion is disappearance of previously noted calcifications.

\section{Extra osseous Chondrosarcoma}

It occurs in $3^{\text {rd }}$ to $6^{\text {th }}$ decades. Cricoid cartilage, thyroid cartilage and other soft tissues are targets. A large mass with calcification gives a clue.

\section{Lymphoma - Leukemia}

Two major categories are noted. 1 . Hodgkin's lymphoma and 2. Non Hodgkin's lymphoma.

\section{Hodgkin's lymphoma}

It is encountered in $3^{\text {rd }}$ and 4 th decades. It is more common in males. Four histological sub types are noted. Radiologically, osteolytic lesions occurs in $2 / 3^{\text {rd }}$ of cases. Mixed and sclerotic lesions in 30\% and purely sclerotic lesions $5-10 \%$.

\section{Non-Hodgkin's lymphoma}

It is almost the same age group of Hodgkin's lymphoma and it is more common than the Hodgkin's. Lytic and mixed lesions are more common. Minor periosteal reactions may be noted (fig. 20).

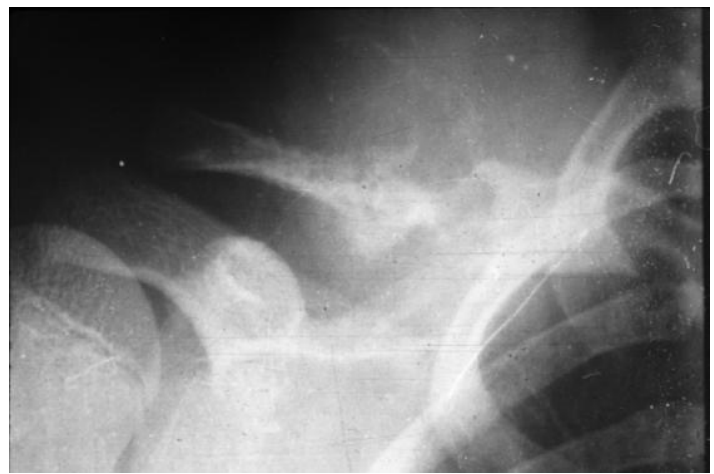

Fig. 20: 12 yr old boy - Histiocytic lymphoma. Mixed lesion of clavicle with pathological fracture

\section{Leukemia}

It is common in childhood. In the first two decades diffuse osteoporosis is noted in the vertebrae with compression fractures. In the long bones horizontal radiolucent bands 
occur in the metaphysis. Multiple small lucencies and moth eaten pattern may be seen with mild periosteal reactions.

In adults it is usually chronic. Generalized osteopenia is noted. In chronic myelogenous and lymphatic leukemias the radiological findings are purely defined. Osteolytic area with mottling is noted particularly in the spine (fig. 21).

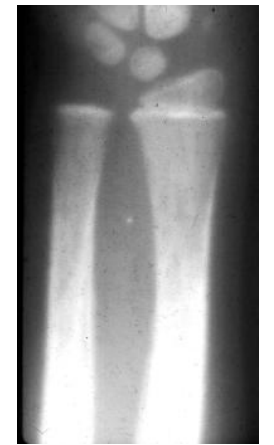

Fig. 21: 6 yr old boy with acute lymphatic leukemia. Note the moth eaten appearance

\section{Fibrosarcoma}

It generally occurs in $3^{\text {rd }}$ to $4^{\text {th }}$ decades. Most of the lessions occur in the lower limbs. Diametaphysis is the common site. Radiologically a lytic moth eaten area is noted in the medullary cavity with wide zone of transition. No calcification or new bone is present. Minor periosteal reaction may be seen.

Malignant fibrous histiocytoma is a histological diagnosis and may develop secondary to bone infarct, Paget's disease or post radiation area. Radiologically, a well defined lytic area with cortical destruction is noted. Occasionally, soft tissue calcification may be seen. Very little periosteal reaction is observed (fig. 22ab).
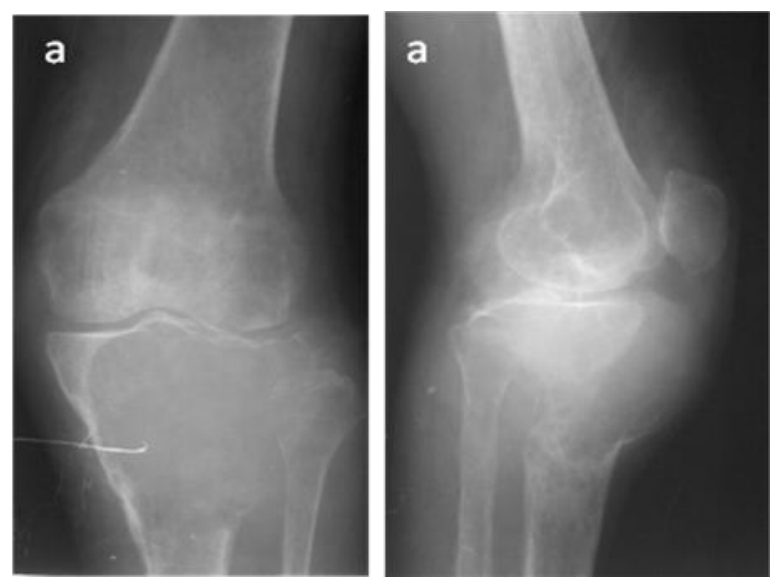

Fig. 22a: Malignant fibrous histiocytoma in the proximal tibia. Large osteolytic lesion extending to the articular margin simulating Giant Cell Tumor
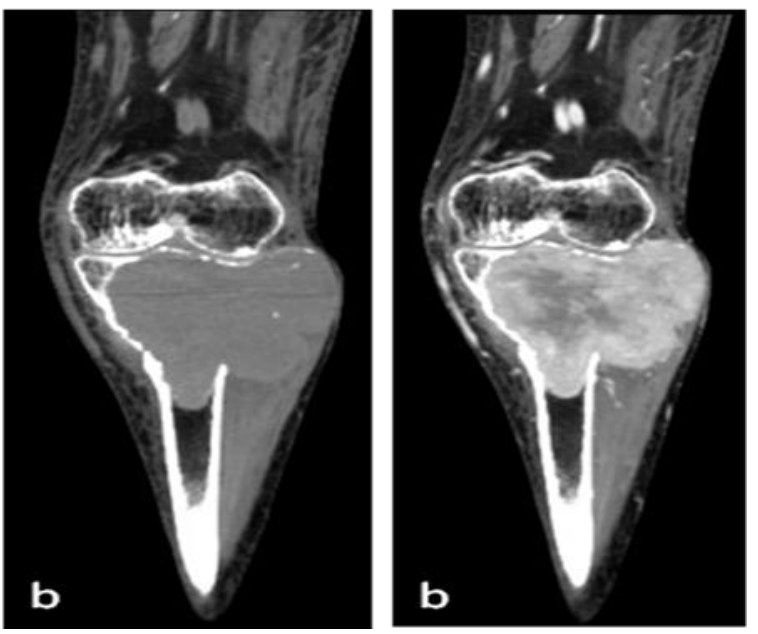

Fig.22b: Malignant fibrous histocytoma in $62 \mathrm{yr}$ old. MRI shows the soft tissue component

\section{Plasmacytoma - Multiple Myeloma}

This is encountered around 50 years of age. It a solitary focus of myeloma. An expanding lytic lesion with wide zone of transitions and a soft tissue mass are characteristic. Spinal lesions are associated with large soft tissue masses. CT and MRI are of help. It often simulates solitary metastasis from carcinoma of the lung, breast and kidney. The patient may present with a pathological fracture (fig. 23, 24ab and 25). 


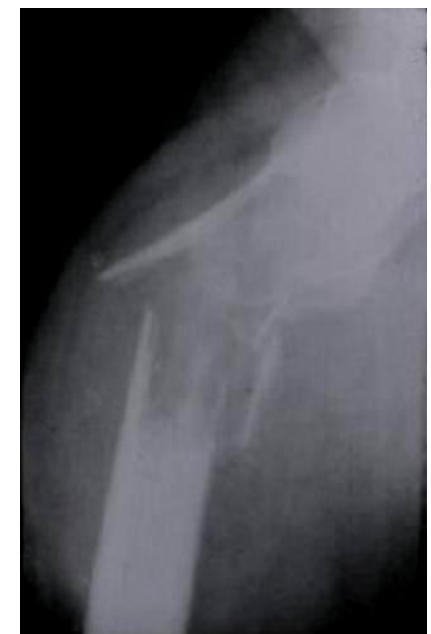

Fig.23: Plasmacytoma of femur with a pathological fracture
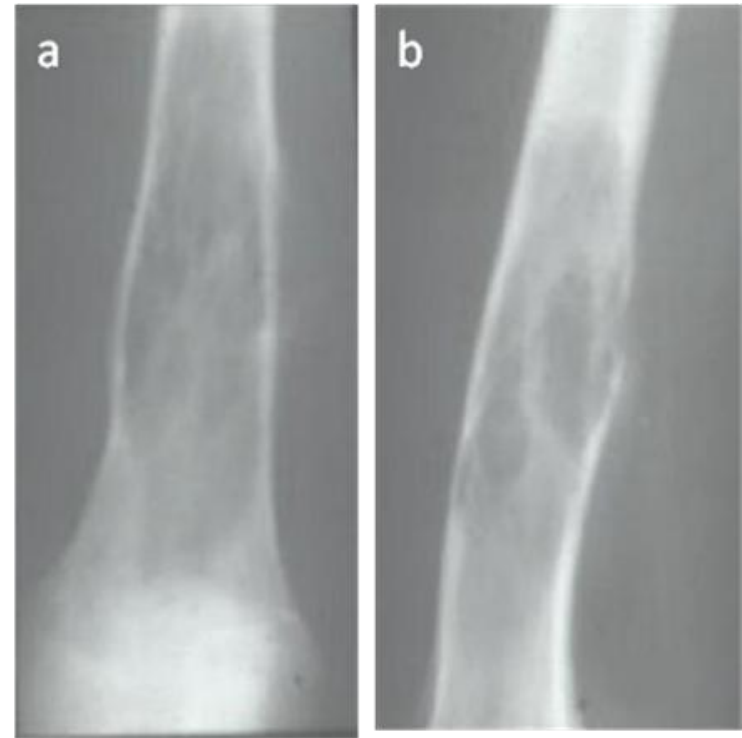

Fig. 24ab: Plasmacytoma of lower end of femur in a 60 yr old

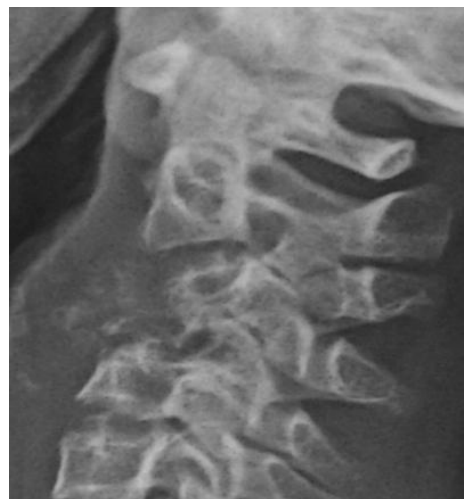

Fig.25 : Plasmacytoma of C3 body with lysis and soft tissue swelling

Multiple myeloma
There are about eight radiological patterns described in the literature. Multiple punched out lesions in the skull are seen only in $30 \%$ of the cases. However, it may be only the radiological finding. Generalized osteopenia with compression of the fracture of the spine are noted. Permeative mottled pattern of lysis is another finding. Some of the lesions may be expanded and some may be small lytic areas. Bence Jones proteins in the urine are noted in about $60 \%$ of the cases (fig. $26 \& 27$ ).

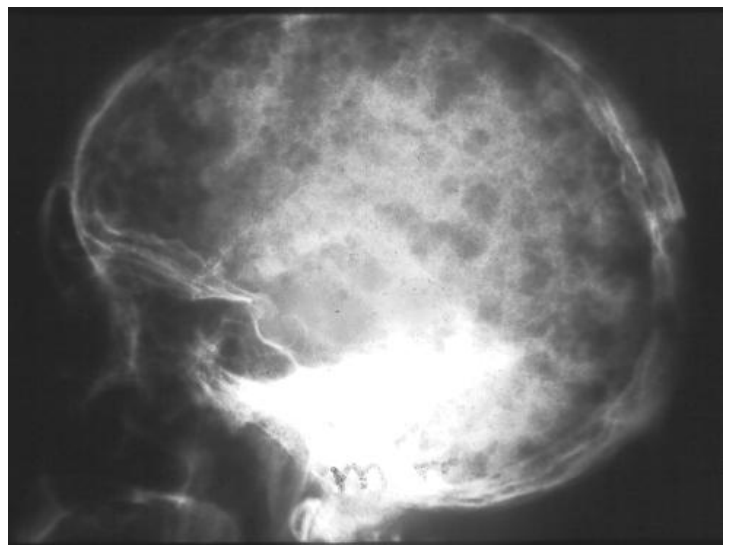

Fig.26: Multiple myeloma. Note the several lytic lesions in the skull

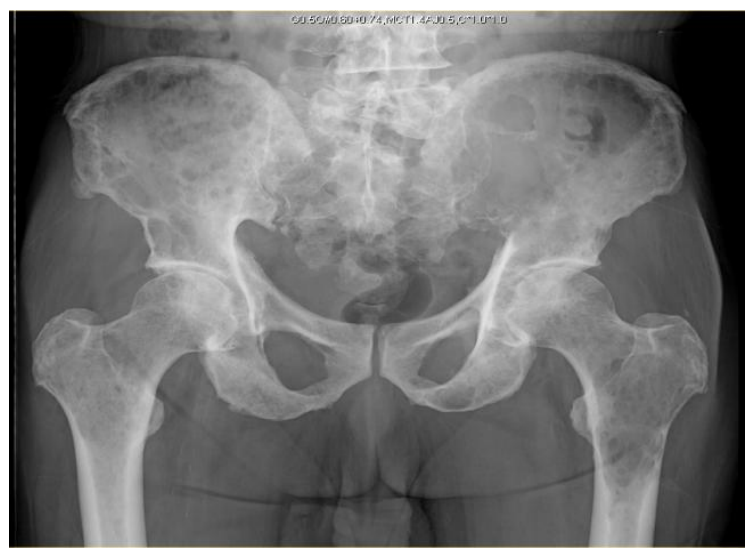

Fig. 27: Multiple myeloma with several osteolytic lesions simulating metastasis

\section{Poems syndrome}

This mnemonic represents ' $\mathrm{P}$ ' for peripheral neuropathy, ' $\mathrm{O}$ ' for organomegaly, ' $\mathrm{E}$ ' for endocrinal changes such as diabetes, ' $M$ ' for myeloma spike and ' $S$ ' for soft tissues changes. 
Radiologically, the bone lesions are osteosclerotic. However, in rare cases osteolytic lesions may also be noted (fig. 28).

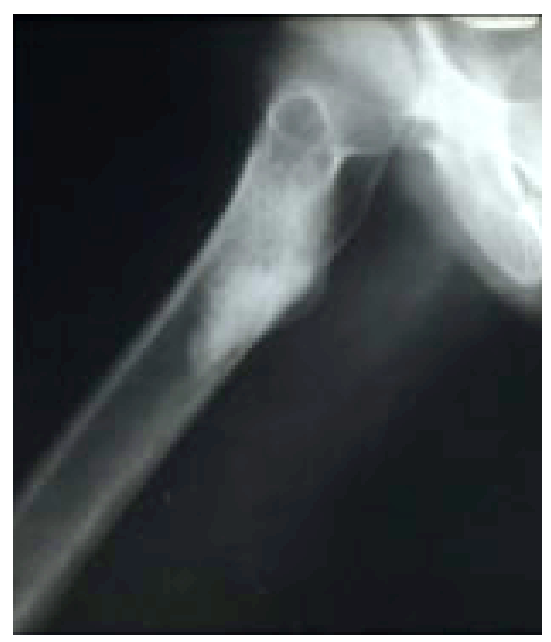

Fig. 28: Poems syndrome - Sclerotic lesion in femur

Giant cell tumors may be malignant de novo are a benign tumor may become malignant. However, benign giant cell tumors may also produce pulmonary metastasis (fig. 29).

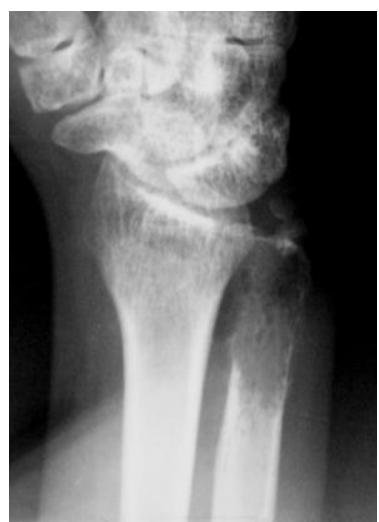

Fig.29: Malignant GCT of lower end of ulna Histological proof
These can be osteolytic, osteosclerotic or a combination of these. The common primaries producing metastasis include carcinoma of the lung, thyroid, breast in females. In males prostate is the most primary site in producing sclerotic metastasis. Mucous secreting adenocarcinoma may produce calcifying or ossifying metastasis. Any soft tissue sarcoma also may produce lytic metastasis.

Gastrointestinal and lower urinary tract lesions produce both lytic and sclerotic metastasis (fig. 30ab). Carcinoids may produce sclerotic metastasis. Sclerotic lesions are listed in table IX.

\section{Osteosclerotic - Table IX}

- Irregular islands of sclerosis

- Axial and peripheral skeleton.

- Primaries include prostate in males and breast in women

- Carcinoid tumors

Mixed type of metastasis may occur in any malignancy from a primary in any organ of the body.

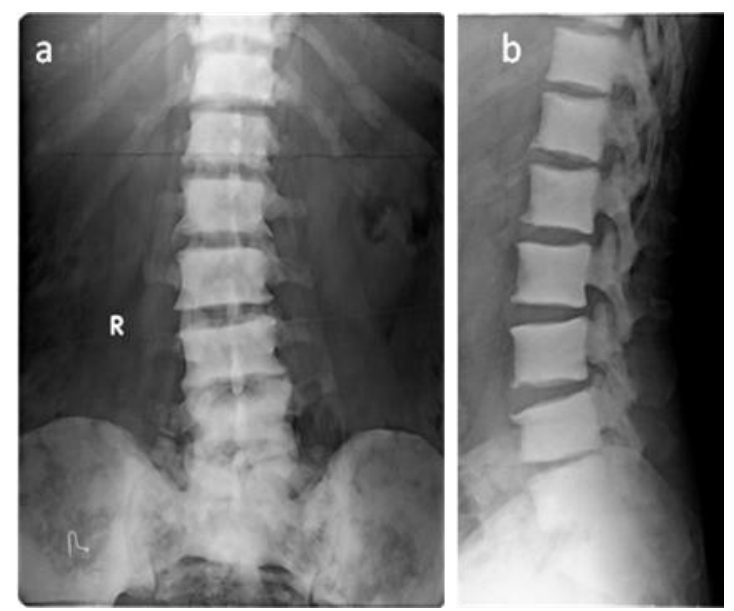

Fig.30ab: Diffuse sclerotic metastasis from carcinoma of the prostate

For the final diagnosis, evidence should be based on histological characteristics Table $\mathbf{X}$

\section{Bone Metastases}




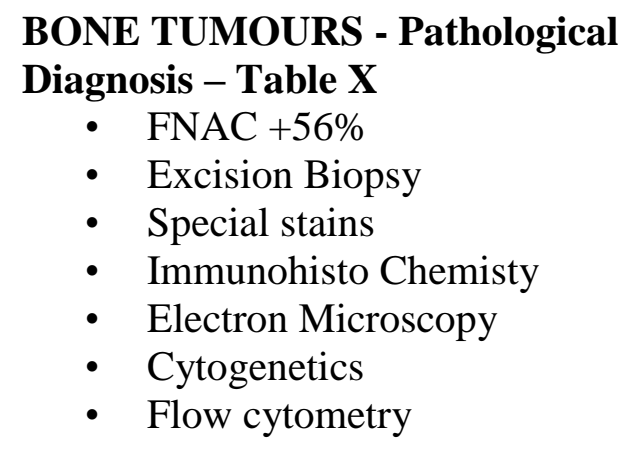

\section{Conclusion}

To sum up primary malignant bone tumors are rare and can be diagnosed by plain films most of the time. However, CT \& MRI would help in further analyzing and malignancy. Finally, histopathology would be the answer. Secondary malignant bone tumors are common and the primary site should be investigated. Rarely, the primary may be unknown.

\section{Further Reading}

1. Edeiken J, Hodes PJ: Roentgen Diagnosis of Disease of Bone Baltimore: Williams and Wilkins, 1967.

2. Enneking WK: Musculoskeletal Tumor Surgery Edinburgh: Churchill Livingstone, 69: 168, 1983.

3. Greenfield GB: Radiology of Bone Diseases (4th ed) Philadelphia JB Lippincott Co. 446-58, 1986.
4. Kyle RA: Multiple myeloma-review of 860 cases. Mayo Clin Proc 50: 29-39, 1975.

5. Meszaros WT: The many facets of multiple myeloma. Semin Roentgenol 9: 219, 1974.

6. Murray RO, Jacobson HG: The Radiology of Skeletal Disorders (2nd ed) London: Churchill Livingstone, 1977.

7. Simon MA, Bartucci EJ: The search for the primary tumor in patients with skeletal matastases of unknown origin. Cancer 58(5): 1088-95, 1986.

8. Simmons CR, Harle TS, Singletion EB: The osseous manifestations of leukemia in children. Radiol C/in North Am 6: $115,1968$.

9. Subbarao K, Jacobson HG: Primary malignant neoplasms. Semin RoentgenoI 14(1): 44-57, 1979.

10. VANEL D. et al. The Radiological appearances of Telangiectatic Osteosarcoma (14 cases). Skeletal Radiol (1987) $16: 196-200$. 